\title{
Anti-oxidative effects of nicotinamide mononucleotide, a regulator of aging, on rat high glucose-induced tenocytes in vitro
}

Kohei Yamaura ( $\nabla$ koheidesuyo@yahoo.co.jp )

Kobe University Graduate School of medicine https://orcid.org/0000-0003-2878-1390

\section{Yutaka Mifune}

Kobe University Graduate School of Medcine

Atsuyuki Inui

Kobe University Graduate School of Medicine

Hanako Nishimoto

Kobe University Graduate School of medicine

\section{Takeshi Kataoka}

Kobe University Graduate School of Medicine

\section{Takashi Kurosawa}

Kobe University Graduate School of Medicine

Shintaro Mukohara

Kobe University Graduate School of Medicine

Takahiro Niikura

Kobe University Graduate School of Medicine

\section{Ryosuke Kuroda}

Kobe University Graduate School of Medicine

\section{Research article}

Keywords: High-glucose, Oxidative stress, Nicotinamide mononucleotide, NADPH oxidase, SIRT

Posted Date: July 22nd, 2020

DOl: https://doi.org/10.21203/rs.3.rs-44917/v1

License: (c) (1) This work is licensed under a Creative Commons Attribution 4.0 International License. Read Full License 


\section{Abstract}

Background Nicotinamide adenine dinucleotide $\left(\mathrm{NAD}^{+}\right)$plays an important role in energy metabolism, mitochondrial function, aging, and cell death. Nicotinamide mononucleotide (NMN) is one of the key precursors of $\mathrm{NAD}^{+}$. The purpose of this study is to evaluate the oxidative stress effects of NMN on rat tenocytes in-vitro.

Methods Tenocytes from normal Sprague-Dawley rats were cultured in regular glucose (RG) and highglucose $(\mathrm{HG})$ conditions with or without NMN, and were divided into four groups: RG $\mathrm{NMN}^{-}, \mathrm{RG} \mathrm{NMN}^{+}, \mathrm{HG}$ $\mathrm{NMN}^{-}$, and $\mathrm{HG} \mathrm{NMN}{ }^{+}$. Cell viability, reactive oxygen species (ROS) production, apoptosis, and messenger RNA (mRNA) expressions of NADPH oxidase (NOX) 1, NOX4, interleukin (IL)-6, SIRT1, and SIRT6, were determined in-vitro.

Results The NMN groups led to significantly higher cell viabilities compared with the other groups. The mRNA expressions of NOX1, NOX4, and IL6, in the HG NMN ${ }^{+}$group were significantly lower compared with those of the HG NMN ${ }^{-}$group. Conversely, the corresponding expressions of the SIRT1 and SIRT6 levels in the $\mathrm{HG} \mathrm{NMN}^{+}$group were significantly higher compared with those of the $\mathrm{HG} \mathrm{NMN}^{-}$group. Both the accumulation of ROS and apoptosis in the $\mathrm{HG} \mathrm{NMN}^{-}$group were significantly higher compared with those in the RG NMN${ }^{-}$group at $48 \mathrm{~h}$.

Conclusion The expression levels of NOX1, NOX4, IL6, and ROS were significantly reduced by NMN. These results suggest that NMN could effectively reduce the oxidative stress by activating SIRT1 and SIRT6, and by inhibiting the activity of NOX and apoptosis in the tenocytes.

\section{Introduction}

Musculoskeletal disorders of the hand and shoulder are more common in diabetic than nondiabetic patients [1]. Diabetes mellitus is associated with many musculoskeletal disorders, such as tendinitis, joint stiffness, tendon ruptures, carpal tunnel syndrome, Dupuytren's disease, and adhesive capsulitis $[2,3,4,5$, $6,7]$. An in vivo study showed hyperglycemia-impaired tendon-bone healing in a rat model of a rotator cuff tear [8]. Another study showed that patients with rotator cuff tears had significantly higher fasting plasma glucose levels within the normoglycemic range than patients with meniscal tears, and concluded that increasing plasma glucose levels may be a risk factor in rotator cuff tear cases [9].

The suggested pathogenesis is related to the excessive generation of oxidative stress caused by hyperglycemia [8]. Oxidative stress induced by hyperglycemia is triggered by reactive oxygen species (ROS) and is controlled by antioxidant enzymes, such as superoxide dismutase and catalase. Accordingly, increases in ROS cause both deoxyribonucleic acid (DNA), ribonucleic acid (RNA), and protein damages, while alterations in antioxidant enzyme levels lead to cellular and tissue damages and to organ dysfunction $[10,11,12,13]$. Other previously published studies reported that the main source of ROS was NADPH oxidase (NOX) and its activation increased ROS production $[14,15,16]$. 
Nicotinamide adenine dinucleotide $\left(\mathrm{NAD}^{+}\right)$was discovered more than a century ago as a low-molecular weight substance in boiled yeast extracts, and was shown to be capable of stimulating fermentation and alcohol production in-vitro [17]. Recently, it has become clear that the cellular role of NAD ${ }^{+}$extends far beyond its classic participation in redox reactions given that it also acts as a substrate for several families of regulatory enzymes $[18,19,20,21]$. A number of studies have demonstrated that $\mathrm{NAD}^{+}$ declines during the aging process and plays an important role in energy metabolism, mitochondrial function, aging, and cell death $[22,23,24]$. One of the key precursors of $\mathrm{NAD}^{+}$is nicotinamide mononucleotide $(\mathrm{NMN})$ that is converted to $\mathrm{NAD}^{+}$by nicotinamide mononucleotide adenylyltransferase [23]. It has been recently shown in various animal models that NMN mitigates age-associated physiological changes in liver, adipose tissue, muscle, pancreas, kidney, and in the central nervous system $[25,26,27,28]$. However, previous studies have not evaluated the antioxidative effect of NMN on tenocytes. The purpose of this study is to evaluate the oxidative stress effects of NMN on rat tenocytes.

\section{Materials And Methods}

All animal procedures were performed in accordance with our approved experimental protocol and the guidance of the Animal Care and Use Committee of our institution.

\section{Cell culture}

Sprague-Dawley (SD) female rats (age = eight weeks) were used in this study. Achilles tendons were excised from SD rats and were washed twice with phosphate buffered saline (PBS). Tissues were cut in small pieces with approximate sizes in the range of 1.5-2.0 $\mathrm{mm}^{3}$. Several pieces were placed on a culture plate and cultured in Dulbecco's Modified Eagle's Medium (DMEM, HyClone, Logan, UT, USA) mixed with $10 \%$ fetal bovine serum (FBS, Cansera, Rexdale, Ontario, Canada), $100 \mathrm{U} / \mathrm{ml}$ penicillin, and $100 \mu \mathrm{g} / \mathrm{ml}$ streptomycin. The explants were incubated at $37^{\circ} \mathrm{C}$ in a humidified atmosphere of $5 \%$ $\mathrm{CO}_{2} / 95 \%$ air. The cells from the tendons were subcultured after trypsin digestion. The media were changed every five days. In this study, cells at passage 2 were used.

\section{Cell proliferation assay}

Cell proliferation was measured by a water-soluble tetrazolium salt (WST) assay with a cell counting kit-8 (Dojindo, Kumamoto, Japan). All of the wells in the 96-well plates were seeded with 2,000 cells and were filled with $100 \mu \mathrm{LMEM}$. The 96-well plates were then placed in a $\mathrm{CO}_{2}$ incubator at $37^{\circ} \mathrm{C}$ before the WST assay evaluation. The cells were exposed to DMEM in regular glucose (RG) concentrations (12 $\mathrm{mM})$ with four different NMN concentrations $(0,10 \mu \mathrm{M}, 100 \mu \mathrm{M}$, and $1 \mathrm{mM})$ for $48 \mathrm{~h}$. For the WST assay, each well was supplemented with $10 \mu \mathrm{l}$ of WST for $4 \mathrm{~h}$ at $37^{\circ} \mathrm{C}$ in a $\mathrm{CO}_{2}$ incubator before spectrophotometric evaluation. The conversion of WST to formazan was measured at $450 \mathrm{~nm}$ spectrophotometrically.

\section{Experimental protocol}


Tenocytes were seeded in twelve-well culture plates at a density of $1 \times 10^{5}$ cells per well. These were then incubated in DMEM at two different glucose concentrations, namely, at $12 \mathrm{mM}$ in the regular glucose (RG) group and at $33 \mathrm{mM}$ in the high-glucose (HG) group without FBS to avoid overgrowth. NMN (Oriental Yeast Co, Tokyo, Japan) was dissolved in PBS, and the final concentration of NMN was $100 \mu \mathrm{M}$ according to the cell proliferation assay outcome. In brief, tenocytes were divided into four groups: a) RG group without NMN $\left(\mathrm{RG} \mathrm{NMN}^{-}\right)$, b) RG group with NMN $\left.\left(\mathrm{RG} \mathrm{NMN}^{+}\right), \mathrm{c}\right) \mathrm{HG}$ group without NMN (HG $\left.\mathrm{NMN}^{-}\right)$, and d) $\mathrm{HG}$ group with NMN ( $\left.\mathrm{HG} \mathrm{NMN}^{+}\right)$.

\section{Quantitative reverse transcription polymerase chain reaction (RT-PCR) analysis}

At $48 \mathrm{~h}$, the total amount of RNA from all tenocytes was extracted with a RNeasy Mini Kit (Qiagen, Valencia, CA). With the use of a high-capacity complementary DNA (CDNA) reverse transcription kit (Applied Biosystems, Foster City, CA), the total RNA was reverse transcribed in a single strand cDNA. Realtime PCR was performed in triplicate on the CDNA with an Applied Biosystems $7900 \mathrm{HT}$ fast real-time PCR system and SYBR Green reagents (Applied Biosystems). Housekeeping gene expression levels were normalized and expressed relative to the control (untreated) culture levels using the $2^{-\Delta \Delta C t}$ method.

\section{ROS measurements}

At $48 \mathrm{~h}$, the accumulation of intracellular ROS levels in tenocytes was detected by the oxidation-sensitive fluorescent probe 2'7'-Dichlorofluorescin Diacetate (DCFH-DA) using the total ROS/Superoxide detection kit (Enzo life Sciences, Farmingdale, New York) according to the manufacturer's protocol. Tenocytes ( $1 \times$ $10^{4}$ ) were incubated with a final DCFH-DA concentration of $10 \mu \mathrm{M}$ for $60 \mathrm{~min}$ at $37^{\circ} \mathrm{C}$ in the dark. Next, they were washed three times with PBS, trypsinized, and resuspended. For quantitative analysis of ROS accumulation, fluorescence intensity was calculated by Adobe Photoshop CC 2020 software (Adobe Systems Incorporated, San Jose, USA) and normalized to cell number as determined by 2-(4amidinophenyl)-1 $1 \mathrm{H}$-indole-6-carboxamidine (DAPI) in randomly selected fields.

\section{Immunofluorescence staining for analysis of apoptotic cells}

Nuclear fragmentation was detected by TUNEL staining with an APO-DIRECT ${ }^{\mathrm{TM}}$ kit (PHOENIX FLOW SYSTEMS, San Diego, CA) with fixed cells (4\% paraformaldehyde/PBS) and DAPI according to the manufacturer's protocol. Apoptotic cells and DAPI-positive cells in four rectangular areas $(0.75 \mathrm{~mm} \times$ $1.0 \mathrm{~mm}$ ) were counted in each slide, and the average values were calculated for quantitative measurements. The percentage of apoptotic cells were calculated using the formula (number of apoptosis-positive nuclei/ number of DAPI-positive nuclei) $\times 100$.

\section{Statistical analyses}

All data were expressed as the mean \pm standard deviation (SD). Cell proliferation was analyzed with oneway analysis of variance (ANOVA). For a comparison of the four groups, two-way ANOVA was performed. 
Post-hoc analyses were performed by the Fisher's protected least-significant difference test. A p-value < 0.05 was considered statistically significant.

\section{Results}

\section{Cell proliferation assay}

Cell proliferation in the groups with $\mathrm{NMN}(10 \mu \mathrm{M}, 100 \mu \mathrm{M}$, and $1 \mathrm{mM})$ was significantly higher than that in the groups without NMN (Fig. 1). The proliferation in the NMN group $(100 \mu \mathrm{M})$ was the highest among all groups. However, there were no significant differences in the three groups with NMN. Thus, the middle concentrations $(100 \mu \mathrm{M})$ were selected in the following experiments.

\section{Quantitative RT-PCR}

The mRNA expressions of NOX1, NOX4, and IL6 in the HG NMN' groups were significantly higher than those in $\mathrm{RG} \mathrm{NMN}^{-}$group at $48 \mathrm{~h}$ (Fig. 2). The mRNA expressions of these markers in the $\mathrm{HG} \mathrm{NMN}^{+}$ groups were significantly lower compared to those in the HG NMN'- group. Conversely, the mRNA expressions of SIRT1 and SIRT6 in the HG NMN ${ }^{-}$groups were significantly higher than those in the RG $\mathrm{NMN}^{-}$group. The mRNA expressions of these markers in the $\mathrm{HG} \mathrm{NMN}^{+}$groups were significantly higher compared with those in the $\mathrm{HG} \mathrm{NMN}^{-}$group.

\section{ROS analyses}

The intracellular ROS levels were detected using DCFH-DA staining. Fluorescence staining showed ROS accumulation (green) in tenocytes and DAPI (blue) (Fig. 3). The accumulation of intracellular ROS levels in the $\mathrm{HG} \mathrm{NMN}^{-}$group was significantly larger compared to those in the $\mathrm{RG} \mathrm{NMN}^{-}$group at $48 \mathrm{~h}$ (Fig. 4). The accumulation in the $\mathrm{HG} \mathrm{NMN}{ }^{+}$group was significantly smaller compared to that in the $\mathrm{HG} \mathrm{NMN}^{-}$ group, but there was no difference within the RG groups.

\section{Apoptotic cell analyses}

Fluorescence staining showed abnormal nuclear morphology, such as nuclear fragmentation in apoptotic cells (green) and DAPI (blue) (Fig. 5). The numbers of apoptotic cells in the $\mathrm{HG} \mathrm{NMN}^{-}$group were higher compared with those in the RG NMN' group at $48 \mathrm{~h}$, but there were no differences between the RG $\mathrm{NMN}^{-}$ group and $\mathrm{HG} \mathrm{NMN}{ }^{-}$group. The apoptotic cells in the $\mathrm{HG} \mathrm{NMN}^{+}$group were significantly lower compared with those in the $\mathrm{HG} \mathrm{NMN}^{-}$group (Fig. 6).

\section{Discussion}

It has been previously suggested that diabetes mellitus increases susceptibility to tendinopathy $[29,30]$. The previously published reports have suggested several pathological mechanisms regarding tendon 
lesions with diabetes $[2,3,4,5]$, but the molecular mechanisms underlying tendinopathy are still unknown. One report has described that $\mathrm{HG}$ concentrations upregulate the expressions of matrix metalloproteinases (MMPs) in tenocytes. Furthermore, the potential combination of increased local matrix degradation evoked by enhanced MMP expressions and decreased ECM may predispose patients with diabetes to tendinopathy or tendon injuries [31]. Another report showed that immunohistochemistry analyses identified both the higher density of type 1 collagen and an increase in the expression of vascular endothelial growth factor, and increased immunostaining for NFKB p50 nuclear localization in the nucleus in the Achilles tendons of the diabetic group [32].

Furthermore, a number of studies have demonstrated that hyperglycemic conditions induce oxidative stress and cytokine production, and they lead to inflammation and tissue damage in various organs [33, $34,35]$. Ueda et al. reported the upregulation of the expression of mRNA for NOX1 and IL-6, and the production of ROS in HG conditions in tenocytes of the Achilles tendons in rats [36]. Previously published reports showed the main source of ROS is NOX and its activation increased ROS production $[14,15,16]$. It has also been shown that HG levels stimulate ROS production through protein kinase C-dependent activation of NOX in cultured aortic smooth muscle cells and in endothelial cells [37]. In addition, mitochondrial dysfunction caused by hyperglycemia leads to cell apoptosis [38]. In the present study, the expressions of NOX1, NOX4, and IL-6, ROS production, and cell apoptosis, were significantly higher in HG compared with RG conditions. These results are in agreement with those reported previously.

Cumulative evidence has suggested that $\mathrm{NAD}^{+}$plays significant roles in a variety of biological processes, including energy metabolism, mitochondrial functions, calcium homeostasis, antioxidation/generation of oxidative stress, gene expression, immunological functions, aging, and cell death [23]. NMN is a major precursor of $\mathrm{NAD}^{+}$in the salvage pathway of $\mathrm{NAD}^{+}$synthesis where it is converted to $\mathrm{NAD}^{+}$in cells by nicotinamide mononucleotide adenyly|transferase. These findings strongly suggest that enhancing $\mathrm{NAD}^{+}$ biosynthesis by administering NMN is an efficient therapeutic intervention against many disease conditions [39]. The enhancement of $\mathrm{NAD}^{+}$leads to the upregulation of key $\mathrm{NAD}^{+}$-consuming enzymes, such as sirtuins, poly-adenosine diphosphate-ribose polymerases, and CD38/157 ectoenzymes that play critical roles in many biological processes $[23,40]$. In the present study, the administration of NMN upregulated significantly cell proliferation and increased significantly the expressions of SIRT1 and SIRT6 in $\mathrm{HG}$ conditions.

Many studies have demonstrated that SIRT1 is a potent intracellular inhibitor of oxidative stress and inflammatory responses $[41,42]$. SIRT1 regulates immune responses via NF-KB signaling. The NF-KB signaling is a crucial regulator of the immune defense system and an inducer of inflammatory responses [43]. The NF-KB system is also involved in many housekeeping and survival functions during cellular stress by controlling apoptosis, proliferation, and energy metabolism [44, 45]. Yeung et al. reported that SIRT1 could inhibit the transactivation capacity of the NF-KB complex by deacetylating the Lys 310 residue of the RelA/p65 component [46]. NF-KB signaling is a potent inducer of the expression of NOX components, such as gp91 phox and p22 phox [47]. The present study showed that the administration of NMN reduced significantly the expressions of NOX1, NOX4, and IL-6, in HG conditions. Quantitative 
analysis of ROS production also showed that treatment with NMN reduced ROS production in HG conditions. These results showed that inhibition of the activity of NOX led to reduced ROS production.

SIRT6 is associated with diverse enzymatic activities, including deacetylation and ribosylation [48]. SIRT6-dependent deacetylation is essential to the regulation of DNA repair, cellular glucose/lipid metabolism, telomere maintenance, cellular senescence, and life span [49]. Fan et al. showed that SIRT6 alleviated HG induced podocyte apoptosis by activating $5^{\prime}$ adenosine monophosphate protein kinase (AMPK) [50]. Furthermore, the previous report showed that the overproduction of ROS causes cellular damage and promotes the process of apoptosis based on the activation of caspase and the regulation of the expressions of the Bcl-2 family proteins [51]. In the present study, the quantitative analysis of apoptosis showed that treatment with NMN reduced apoptosis in HG conditions.

There are several limitations associated with this study. Firstly, the in-vitro results were preliminary. Therefore, clinical applications in humans will require additional detailed research including in vivo animal studies. Secondly, the monolayer culture of tenocytes in-vitro never reproduced the actual physiological conditions. Previous studies have demonstrated that primary tenocytes maintained the phenotypical stability until passage 5 when the cultures in prior passages were in subconfluent states [52]. In the present experiments, tenocytes were cultured after passage 2. Finally, while there are several apoptotic pathways involved in HG conditions, the mediator of the apoptotic signaling pathways was not investigated in this study.

In conclusion, HG concentrations upregulated the mRNA of NOX1, NOX4, and IL-6 expressions, and the production of ROS and apoptosis. NMN significantly reduced ROS production and cell death based on the activation of SIRT1 and SIRT6 and the inhibition of the activity of NOX. NMN is thus a potential prodrug in the treatment of diabetic tendinopathy.

\section{Abbreviations}

ROS

reactive oxygen species, NOX:NADPH oxidase

NAD

Nicotinamide adenine dinucleotide, NMN:Nicotinamide mononucleotide

SD

Sprague-Dawley, PBS; phosphate buffered saline

DMEM

Dulbecco's Modified Eagle's Medium, FBS:fetal bovine serum

\section{Declarations}

\section{Ethics approval and consent to participate}


The research protocol was approved by the Institutional Animal Care and Use Committee and carried out according to the Kobe University Animal Experimentation Regulations (Permission number P190204).

\section{Consent for publication}

Not applicable.

\section{Availability of data and materials}

The datasets used and/or analyzed during the current study are available from the corresponding author on reasonable request.

\section{Competing interests}

The authors declare that they have no competing interests.

\section{Funding}

Funding information is not applicable.

\section{Authors' contributions}

YM and Al contributed to the conception and design of the study. TKA, TKU and SM performed the experiments and collected the data. Data and statistical analysis was done by YM and Al. Manuscript preparation was done by YM, Al and TN. Supervising was done by RK. All authors read and approved the final manuscript.

\section{Acknowledgements}

The authors would like to thank all participants of this study and Enago (www.enago.jp) for the English language review.

\section{References}

1. Cagliero E, Apruzzese W, Perlmutter GS, Nathan DM. Musculoskeletal disorders of the hand and shoulder in patients with diabetes mellitus. Am J Med. 2002;112:487-90. 
2. Balci N, Balci MK, Tuzuner S. Shoulder adhesive capsulitis and shoulder range of motion in type II diabetes mellitus: association with diabetic complications. J Diabetes Complications. 1999;13:135-40.

3. Mavrikakis ME, Drimis S, Kontoyannis DA, Rasidakis A, Moulopoulou ES, Kontoyannis S. Calcific shoulder periarthritis (tendinitis) in adult onset diabetes mellitus: a controlled study. Ann Rheum Dis. 1989;48:211-4.

4. Pal B, Anderson J, Dick WC, Griffiths ID. Limitation of joint mobility and shoulder capsulitis in insulin- and noninsulin-dependent diabetes mellitus. BrJ Rheumatol. 1986;25:147-51.

5. Ramchurn N, Mashamba C, Leitch E, Arutchelvam V, Narayanan K, Weaver J, et al. Upper limb musculoskeletal abnormalities and poor metabolic control in diabetes. Eur J Intern Med. 2009;20:718-21.

6. Pourmemari MH, Shiri R. Diabetes as a risk factor for carpal tunnel syndrome: a systematic review and metaanalysis. Diabet Med. 2016;33:10-16.

7. Noble J, Heathcote JG, Cohen H. Diabetes mellitus in the aetiology of Dupuytren's disease. J Bone Joint Surg [Br]. 1984;66-B:322-25.

8. Bedi A, Fox AJ, Harris PE, Deng XH, Ying L, Warren RF, Rodeo SA. Diabetes mellitus impairs tendon-bone healing after rotator cuff repair. J Shoulder Elbow Surg 2010;19:978-88.

9. Longo UG, Franceschi F, Ruzzini L, Spiezia F, Maffulli N, Denaro V. Higher fasting plasma glucose levels within the normoglycaemic range and rotator cuff tears. BrJ Sports Med. 2009;43:284-7.

10. Kisic B, Miric D, Dragojevic I, Rasic J, Popovic L. Role of myeloperoxidase in patients with chronic kidney disease. Oxid Med Cell Longev 2016;2016:1069743.

11. Liang W, Zhao YJ, Yang H, Shen LH. Effects of antioxidant system on coronary artery lesions in patients with abnormal glucose metabolism. Aging Clin Exp Res 2016;29:141-6.

12. Aybek H, Aybek Z, Rota S, Sen N, Akbulut M. The effects of diabetes mellitus, age, and vitamin E on testicular oxidative stress. Fertil Steril. 2008;90:755-60.

13. Portal-Nunez S, Ardura JA, Lozano D, Martinez de Toda I, De la Fuente M, Herrero-Beaumont G, et al. Parathyroid hormone-related protein exhibits antioxidant features in osteoblastic cells through its N-terminal and osteostatin domains. Bone Joint Res. 2018;7:58-68.

14. Jin L, Lagoda G, Leite R, Webb RC, Burnett AL. NADPH oxidase activation: a mechanism of hypertensionassociated erectile dysfunction. J Sex Med. 2008;5:544-51. 
15. Jin L, Burnett AL. NADPH oxidase: recent evidence for its role in erectile dysfunction. Asian J Androl. 2008;10:6-13.

16. Cai H, Griendling KK, Harrison DG. The vascular NAD(P)H oxidases as therapeutic targets in cardiovascular diseases. Trends Pharmacol Sci. 2003;24:471-8.

17. Berger F, Ramírez-Hernández $\mathrm{MH}$, Ziegler $\mathrm{M}$. The new life of a centenarian: signalling functions of $\mathrm{NAD}(\mathrm{P})$. Trends Biochem Sci. 2004;29:111-8.

22. Ying W. NAD ${ }^{+}$and NADH in cellular functions and cell death. Front Biosci 2006;11:3129-3148.

23. Ying W. $\mathrm{NAD}^{+} / \mathrm{NADH}$ and $\mathrm{NADP}^{+} / \mathrm{NADPH}$ in cellular functions and cell death: regulation and biological consequences. Antioxid Redox Signal. 2008;10:179-206.

24. Xia W, Wang Z, Wang Q, Guest J, Farnsworth B, Guillemin GJ. Roles of NAD(+) / NADH and NADP(+) / NADPH in cell death. Curr Pharm Des. 2009;15:12-19.

25. Gomes, AP, Price NL, Ling AJ, Moslehi JJ, Montgomery MK, Rajman L, et al. Declining NAD(+) induces a pseudohypoxic state disrupting nuclear-mitochondrial communication during aging. Cell. 2013;155:1624-38.

26. Massudi, H., Grant, R., Braidy, N., Guest J, Farnsworth B, Guillemin GJ. Age-associated changes in oxidative stress and NAD ${ }^{+}$metabolism in human tissue. PLOS ONE. 2012;7:e42357.

27. Stein, L.R., and Imai, S. Specific ablation of Nampt in adult neural stem cells recapitulates their functional defects during aging. EMBO J. 2014;33:1321-40.

28. Mills KF, Yoshida S, Stein LR, et al. Long-term administration of nicotinamide mononucleotide mitigates ageassociated physiological decline in mice. Cell Metab.2016;24; 795-806.

29. Batista F, Nery C, Pinzur M, et al. Achilles tendinopathy in diabetes mellitus. Foot Ankle Int. 2008;29:498501.

30. Ranger TA, Wong AM, Cook JL, Gaida JE. Is there an association between tendinopathy and diabetes mellitus? A systematic review with meta-analysis. Br J Sports Med. 2016;50:982-9.

31. Tsai WC, Liang FC, Cheng JW, Lin LP, Chang SC, Chen HH, et al. High glucose concentration up-regulates the expression of matrix metalloproteinase-9 and -13 in tendon cells. BMC Musculoskelet Disord. 2013;14:255.

32. de Oliveira RR, Martins CS, Rocha YR, Braga AB, Mattos RM, Hecht F, et al. Experimental diabetes induces structural, inflammatory and vascular changes of Achilles tendons. PloS ONE. 2013;8:e74942. 
33. Baynes JW, Thorpe SR. Role of oxidative stress in diabetic complications: a new perspective on an old paradigm. Diabetes. 1999;48:1-9.

34. Kowluru RA, Engerman RL, Kern TS. Diabetes-induced metabolic abnormalities in myocardium: effect of antioxidant therapy. Free Radic Res. 2000;32:67-74.

35. Nishikawa T, Edelstein D, Du XL, Yamagishi S, Matsumura T, Kaneda Y, et al. Normalizing mitochondrial superoxide production blocks three pathways of hyperglycaemic damage. Nature. 2000;404:787-90.

36. Ueda Y, Inui A, Mifune Y, Sakata R, Muto T, Harada Y, et al. The effects of high glucose condition on rat tenocytes in vitro and rat Achilles tendon in vivo. Bone Joint Res. 2018;7:362-72.

37. Inoguchi T, Li P, Umeda F, Yu HY, Kakimoto M, Imamura M, et al. High glucose level and free fatty acid stimulate reactive oxygen species production through protein kinase C--dependent activation of $N A D(P) H$ oxidase in cultured vascular cells. Diabetes. 2000;49:1939-45.

38. Higgins GC, Coughlan MT. Mitochondrial dysfunction and mitophagy: the beginning and end to diabetic nephropathy? BrJ Pharmacol. 2014;171:1917-42.

39. Imai S and Guarente L. (2014). NAD+ and sirtuins in aging and disease.Trends Cell Biol. 2014;24: 464-71.

40. Canto' C, Menzies K.J, and Auwerx J. NAD(+) metabolism and the control of energy homeostasis: a balancing act between mitochondria and the nucleus. Cell Metab. 2015;22:31-53.

41. Salminen A, Kauppinen A, Suuronen T, Kaarniranta K. SIRT1 longevity factor suppresses NF-kB-driven immune responses: Regulation of aging via NF-kB acetylation? BioEssays. 2008:30:939-42.

42. Salminen A, Hyttinen J.M.T, Kaarniranta K. AMP-activated protein kinase inhibits NF-kB signaling and inflammation: Impact on healthspan and lifespan. J Mol Med. 2011;89:667-6.

43. Vallabhapurapu S, Karin M. Regulation and function of NF-kB transcription factors in the immune system. Annu. Rev. Immunol. 2009;27:693-733.

44. Perkins, N.D. Integrating cell-signalling pathways with NF-kB and IKK function. Nat. Rev. Mol. Cell Biol. 2007;8:49-62.

45. Johnson, R.F.; Perkins, N.D. Nuclear factor-kB, p53, and mitochondria: Regulation of cellular metabolism and the Warburg effect. Trends Biochem. Sci. 2012;37:317-24.

46. Yeung F, Hoberg J.E, Ramsey C.S, et al. Modulation of NF-kB-dependent transcription and cell survival by the SIRT1 deacetylase. EMBO J. 2004;23:2369-80.

Page $11 / 17$ 
47. Anrather J, Racchumi G, Iadecola C. NF-kB regulates phagocytic NADPH oxidase by inducing expression of gp91 phox. J Biol Chem. 2006;281:5657-67.

48. Gil R, Barth S, Kanfi Y, Cohen HY. SIRT6 exhibits nucleosome-dependent deacetylase activity. Nucleic Acids Res. 2013;41:8537-45.

49. Kanfi Y, Naiman S, Amir G, Peshti V, Zinman G, Nahum L, et al. The sirtuin SIRT6 regulates lifespan in male mice. Nature. 2012; 483: 218-21.

50. Fan Y, Yang Q, Yang Y, Gao Z, Ma Y, Zhang L, et al. Sirt6 Suppresses High Glucose-Induced Mitochondrial Dysfunction and Apoptosis in Podocytes through AMPK Activation. Int J Biol Sci. 2019;15:701-713.

51. Kaushal N, Bansal MP. Dietary selenium variation-induced oxidative stress modulates CDC2/cyclin B1 expression and apoptosis of germ cells in mice testis. J Nutr Biochem. 2007;18:553-64.

52. Yao L, Bestwick CS, Bestwick LA, Maffulli N, Aspden RM. Phenotypic drift in human tenocyte culture. Tissue Eng. 2006;12:1843-1849.

\section{Figures}

$$
*<0.05
$$

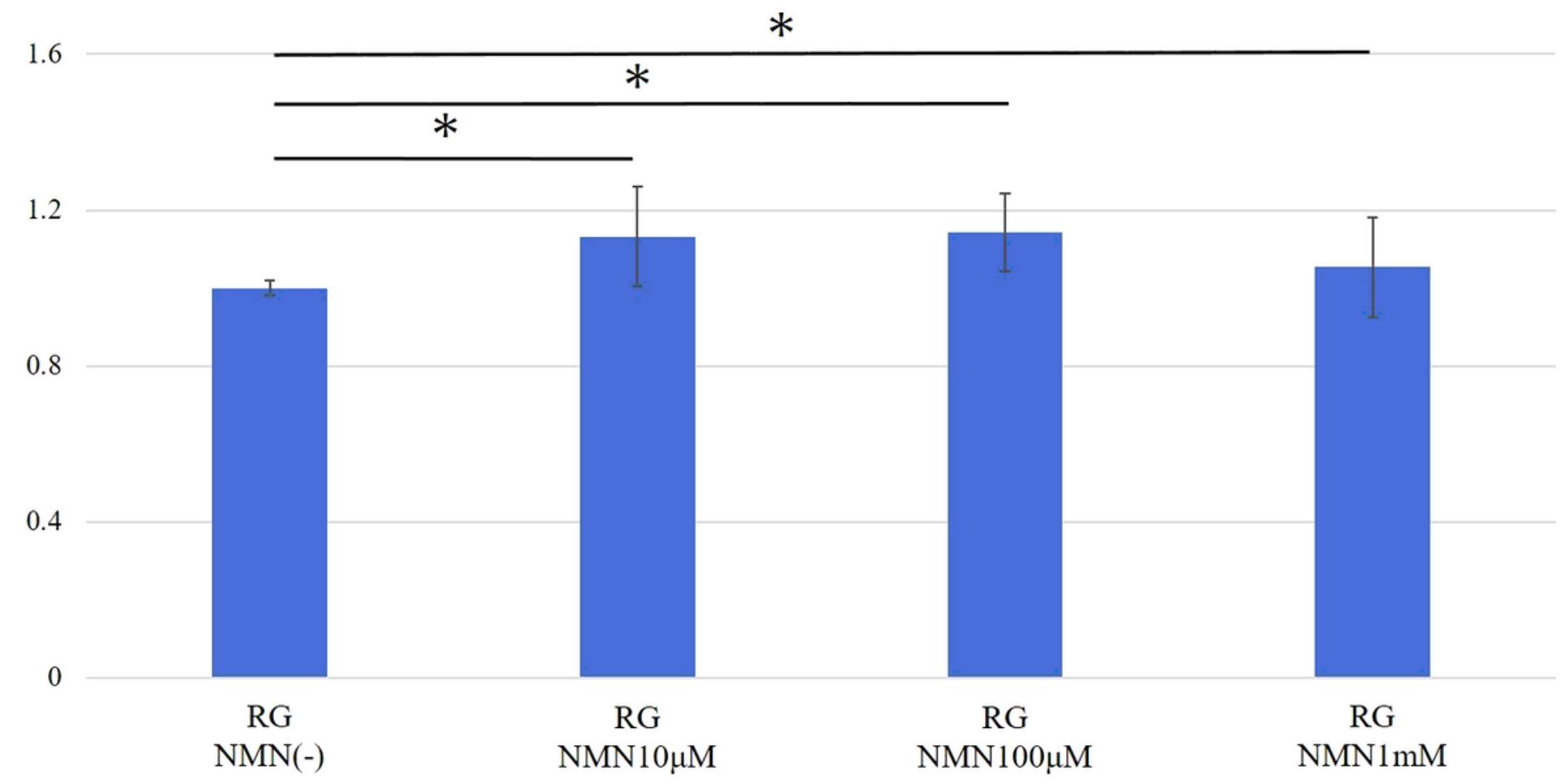

Figure 1 
Cell proliferation Cell proliferation was measured by a water-soluble tetrazolium salt (WST) assay using a cell counting kit-8 (Dojindo, Kumamoto, Japan). The cells were exposed to DMEM in regular glucose concentrations (12 mM) with four different NMN concentrations $(0,10 \mu \mathrm{M}, 100 \mu \mathrm{M}$, and $1 \mathrm{mM})$ for $48 \mathrm{~h}$. Cell proliferations in the groups with NMN $(10 \mu \mathrm{M}, 100 \mu \mathrm{M}$, and $1 \mathrm{mM})$ were significantly higher than that in the groups without NMN ( $\left.{ }^{*} p<0.05\right)$. The proliferation in the group with $100 \mu \mathrm{M} N M N$ was the highest among all groups.
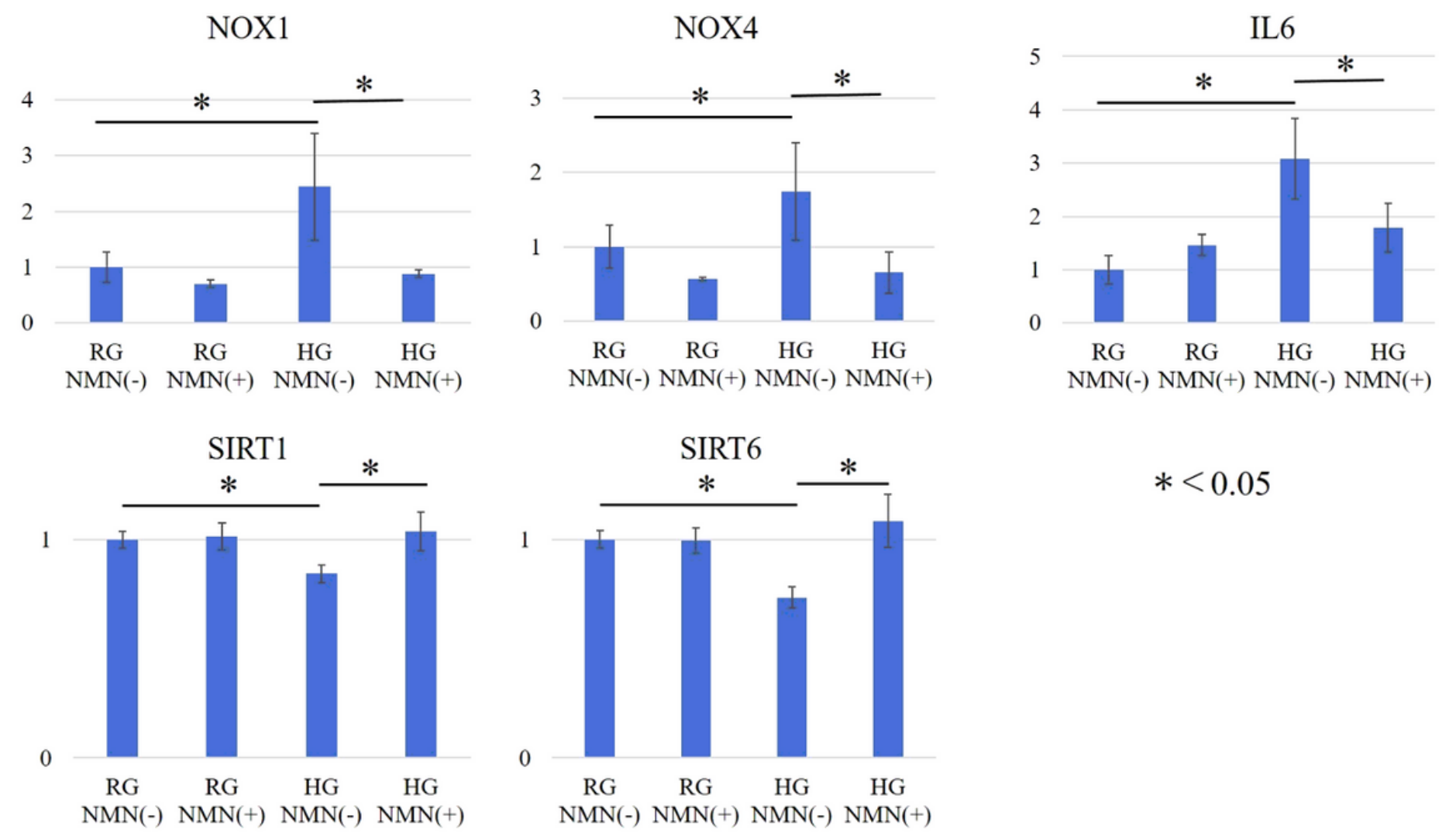

$$
*<0.05
$$

Figure 2

Quantitative reverse transcription PCR The mRNA expressions of NOX1, NOX4, and IL6 in the HG NMNgroups were significantly higher than those in the RG NMN- group at $48 \mathrm{~h}$. The mRNA expressions of these markers in the cases of the HG NMN+ groups were significantly lower compared to those of the HG NMN- group. Conversely, the mRNA expressions of SIRT1 and SIRT6 in the HG NMN- groups were significantly higher than those in the RG NMN-group. The mRNA expressions of these markers in the HG $\mathrm{NMN}+$ groups were significantly higher compared to those of the HG NMN-group. 


\section{RG NMN(-)}

\section{RG NMN(+)}

\section{HG NMN(-)}

\section{HG NMN(+)}

Figure 3

ROS evaluation Intracellular ROS levels were detected with DCFH-DA staining. Fluorescence staining showed ROS accumulation (green) in tenocytes and DAPI (blue). 


\section{$*<0.05$}

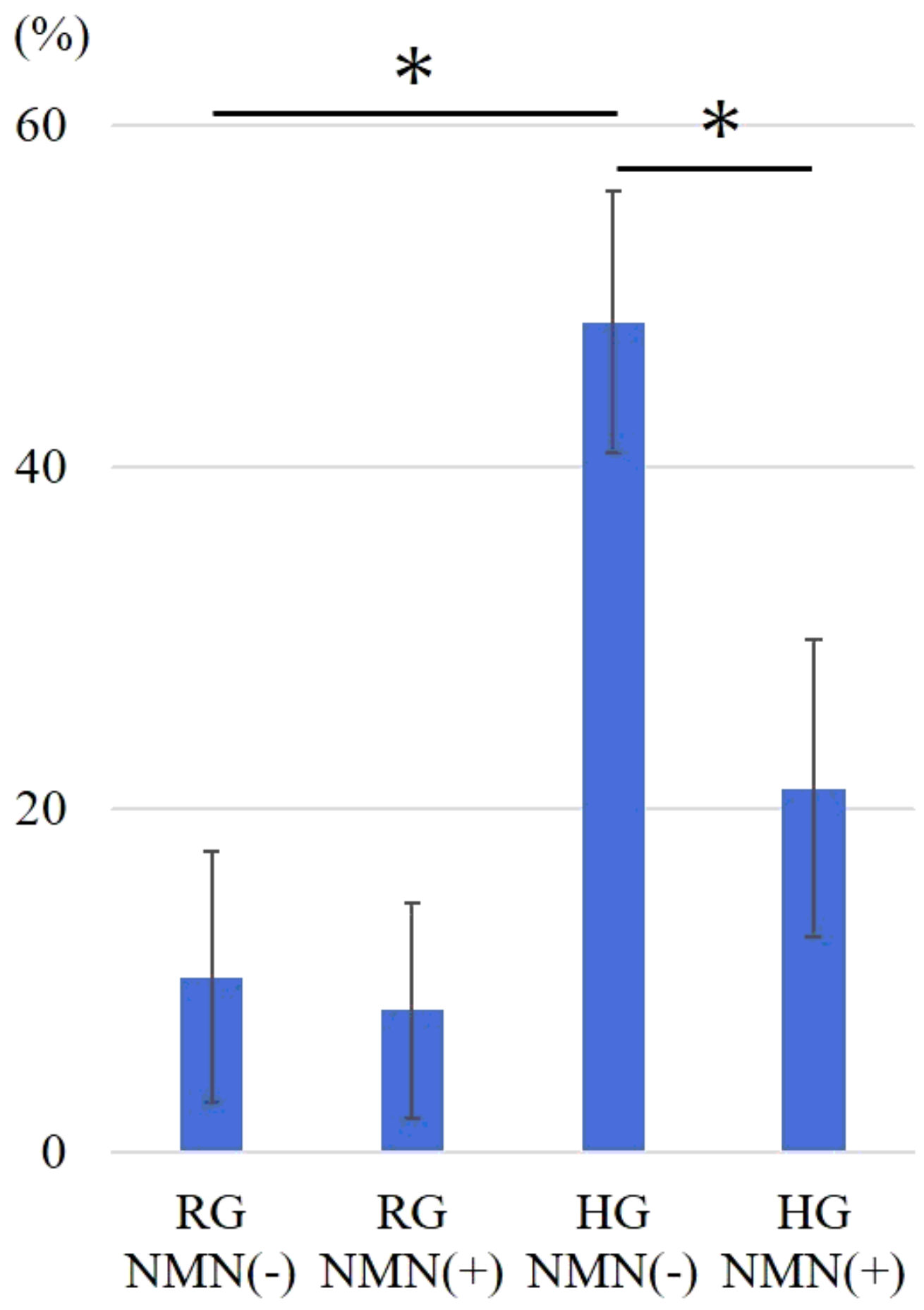

Figure 4

Quantification of ROS accumulation The accumulation of intracellular ROS levels in the HG NMN- group was significantly larger compared with that in the RG NMN-group at $48 \mathrm{~h}$. The accumulation associated with the HG NMN+ group was significantly smaller compared with that of the HG NMN- group, but there was no difference within the RG groups. 


\section{RG NMN(-) \\ RG NMN(+)}

\section{HG NMN(+)}

$\boldsymbol{0}$

8

\section{Figure 5}

Apoptosis examination Fluorescence staining showed abnormal nuclear morphology, such as nuclear fragmentation in apoptotic cells (green) and DAPI (blue). 
$(\%)$

\section{$*<0.05$}
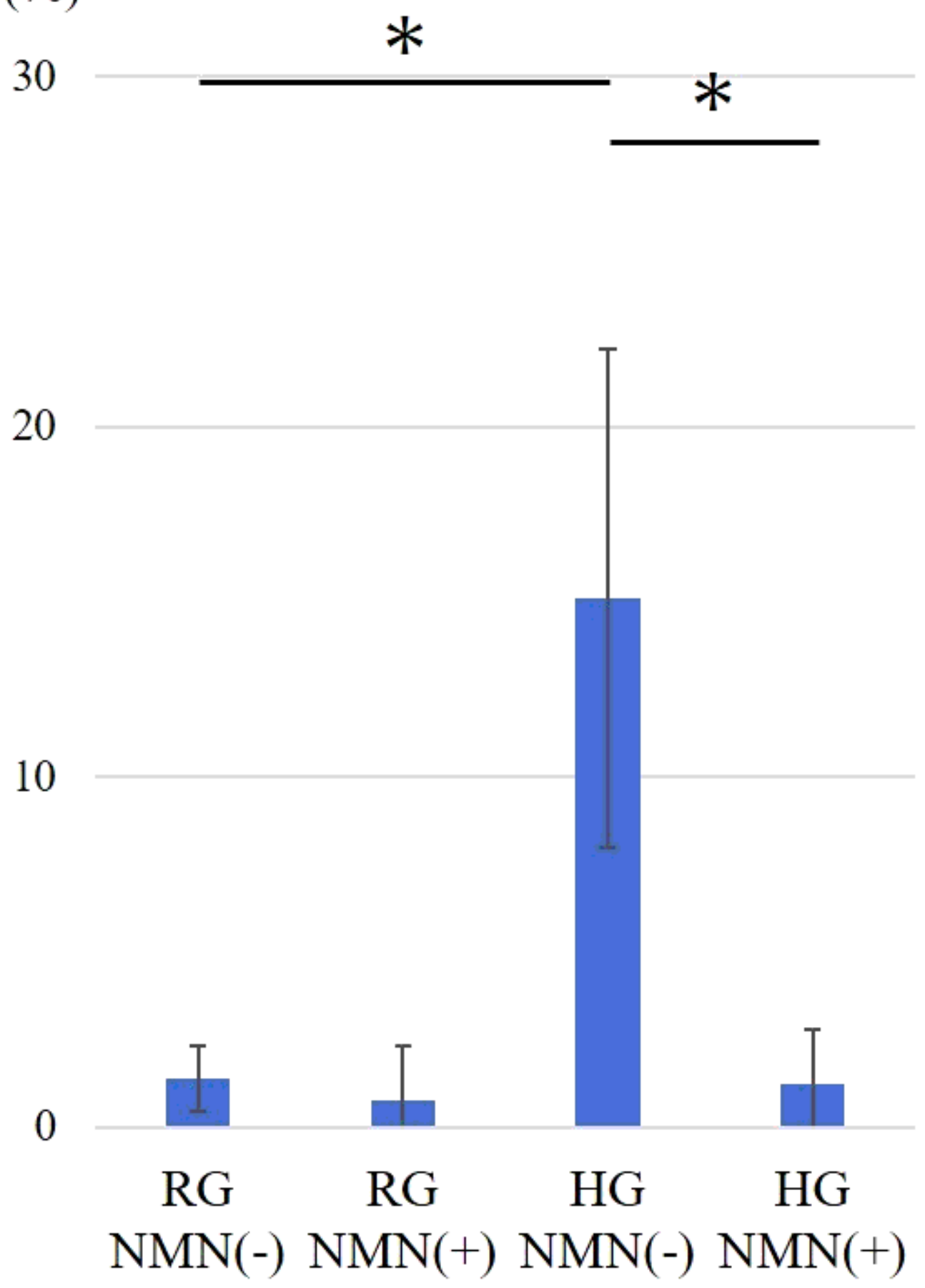

Figure 6

Quantification of apoptotic accumulation Apoptotic cell number associated with the HG NMN-group was higher compared with that of the RG NMN- group at $48 \mathrm{~h}$, but there were no differences between the RG $\mathrm{NMN}$ - and HG NMN- groups. The number of apoptotic cells associated with the HG NMN+ group was significantly lower compared with that of the HG NMN-group. 